\title{
Auditory System Rehabilitation
}

\author{
Available Technologies
}

\author{
Bento, David; Ferreira, Stephanie; Magalhães, Bruno; Rocha, Daniela and Teixeira, João Paulo \\ ESTiG - Polytechnic Institute of Bragança \\ Bragança, Portugal
}

\begin{abstract}
In this article some of the different technologies and its functioning as well as some technological aids for people with partial or total auditory deficiency will be presented. The objective of the auditory rehabilitation is to develop the capacity of auditory perception to the individual carrying auditory deficiency, with aid of devices that can amplify the sound. Between these devices, we cite: the Baha auditory prostheses, vibrant sound-bridge, the cochlear implantations, the auditory brainstem implants, the hearing prosthesis, the bone conduction prostheses and the intra-channels hearing. Some technological aiding devices not used in the ear are also presented such as the signal amplifier to phone, amplifier magnetic field to $\mathrm{TV}$, sign language translator, phone with handset and light bell for home.
\end{abstract}

Keywords-component; hearing; deafness; rehabilitation.

\section{INTRODUCTION}

Auditory deficiency or deafness is the partial or total disability of hearing. It can be caused by born deaf or caused later by illnesses. The hearing impaired person is classified as deaf when its hearing is not functional in the common life and classified as hipoacoustic that someone whose hearing, despite deficient, is functional with or without auditory prosthesis [1, $2,3,4,5]$.

The hearing is an essential sense to life, playing an important role in society, because it is the basis for the development of human communication.

An individual with incapacity hearing can suffer repercussions on their social life, psychological and vocational, emerging also feelings of insecurity, fear, depression, isolation, and tension in the family environment, due to lack the care of patients with hearing impairment [2].

The auditory deficiency can be classified as: transmission deficiency, when the problem is locates in the outer ear or the middle ear; mixing deficiency, when the problem is locates in the middle ear; internal or neuro-sensorial deficiency, when originated in the inner ear or in the auditory nerve [1].

A deaf person is more than somebody with auditory loss. It has a typical culture and unique characteristics that distinguishes it as individual. Its primary language is its sign language [3].

Thus, we cannot see the deaf persons as having only auditory incapacity but as having different abilities of a listener, as the domain of a different language and, therefore one another culture.
It is therefore necessary to recognize these situations, establishing programs of adequate tracings and techniques that help deficient auditory.

The World Health Organization WHO foresees the existence of 42 million deaf people. Although they do not exist accurate statistics, it is foreseen the existence of about 150 thousand deaf people in Portugal [6].

The existing technologies vary in accordance with the type and degree of deafness, the etiologies and the characteristics of the individual. After a study carried through for the team of otorhinolaryngologist, audiologist and speech therapeutic it is determined the aid most efficient.

Periodic consultations and examinations are carried in order to evaluate the evolution of the situation and to adjust the technology to the new conditions that go appearing.

All the auditory devices are projected to increase the volume of the sounds that arrive at the ear canal so that the people with a loss of hearing can understand the speech. The auditory devices require three basic components: a microphone to congregate acoustic energy (waves of sound in the air) and to convert it into electric energy; an amplifier to increase the power of the electric signal and a receiver that is as a miniature loudspeaker that converts again the electric energy into acoustic energy (sound waves).

In next sections we present some available technologies and methodologies used in the rehabilitation of hearing impaired persons described in literature, as a result of an academic work. Section II presents some implant apparatus, section III presents several available hearing devices and section IV other technological aids not connected to the ear.

\section{SOME AVAILABLE IMPLANT DEVICES AND ITS FUNCTIONING}

In this section some auditory implant prosthesis are present.

\section{A. Auditory Prosthesis Osteo Bone cemented (BAHA)}

The Baha is a surgically implantable system for treatment of hearing loss that works through direct bone conduction. They can be adequate for persons with conductive or mixed audivive loss and unilateral deaf. The direct bone conduction is used alowing the cranian bone to transmite the sound to the functional cochlea, not using the outer ear canal or the middle ear bringing also benefits to persons with cronic infections in the ear or estenose of the ear canal [7]. Fig. 1. 


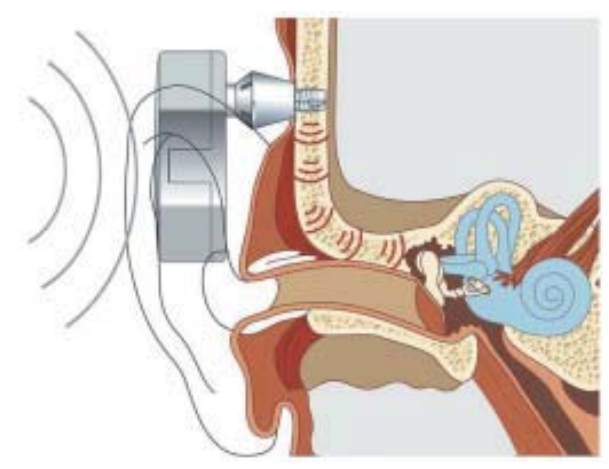

Figure 1. Bone-anchored hearing aids (BAHA) [7].

The implante is placed behind the deaf ear and the vibrations of the implant in response to the sound produce a stimulus to the contralateral ear.

The chirurgical procedure is simple realized in two operations under general anesthesia, separated in time between 3 and 6 months, corresponding to the period of osteo integration of the titanium implant in the bone. Four to six weeks after the second operation the prosthesis is applied given back the audition to the patient [7].

This technique can be applied in the following pathologies:

- Bilateral congenital malformation of the middle ear/external;

- Acquired stenosis of external auditory canal;

- Chronic suppurations of bilateral hearing that does not allow the use of a conventional prosthesis;

- Post status radical bilaterally mastoidectomy;

- Ossicular discontinuity without surgical indication;

- Otosclerosis;

- Chronic external otitis;

- Changes in skin of external auditory channel;

- Unilateral sensorineural hearing loss.

\section{B. Vibrant Soundbridge}

It is a surgically implantable hearing aid in the middle ear, which is an alternative indicated for the treatment of neural hearing loss, mixed or conductive moderate to severe, basically composed of two parts: an external one, called "digital audio processor" and other internal part that is implanted in the ossicles, which transmits the signal, Fig. 2.

This is called Vibrating Ossicular Prosthesis - The general operation of the Vibrant SoundBridge device is based on the sound transmission by audio processor, through the skin to the internal receiver in VORP [8].

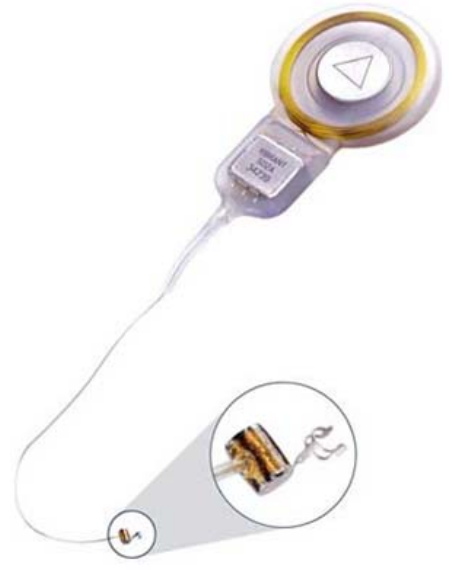

Figure 2. Vibrant Soundbridge [8].

\section{Cochlear Implant}

The cochlear lesion affects a range of abilities of the auditory system, committing the acoustic signal processing or speech and, consequently, the ability of individuals to communicate.

Among these abilities emphasizes selectivity of frequency, the perception of sensation, intensity resolution, temporal resolution and the perception of speech.

The cochlear implant provides electrical stimuli for the stimulation of neural fibers in different regions of the cochlea, allowing the patients the ability to perceive sound.

It was designed to simulate the natural hearing in people who have severe hearing loss. It performs the function of ciliated cells in the cochlea, because it is an electronic hightech (bionic ear) that stimulates electrically nerve fibers, allowing transmission of electrical signals to the auditory nerve in order to be decoded by the brain cortex [9]. Cochlear implants have internal and external components. The internal components consist in a receiver/Stimulator, magnet, an antenna placed under the skin and an electrode placed on scale tympanic of the cochlea. The external components consist in a signal processor (which is usually worn on the belt), a microphone behind the ear and a transmitter which is placed over the mastoid. These components are connected by cables. The transmitter is fixed magnetically above the mastoid to magnet in part deployed device [9]. The microphone behind the ear receives sound and converts it into electrical signals. These signals are sent to external signal processor used in the belt. The signal processor modifies the signal and sends it to the transmitter above the mastoid. The transmitter then sends the signal to the receiver / stimulator implanted directly or indirectly. Directly, the signal can be sent through the cables of a percutaneous connector. Indirectly, the signal can be sent by an FM radio frequency or magnetic induction.

The receiver/Stimulator, deployed under the skin in the mastoid, modifies further signal and sends it to electrodes implanted in the tympanic scale. These electrodes stimulate neural tissue, usually remaining ganglion cells in the cochlea spirals [9]. An exemple is presented in Fig. 3. 


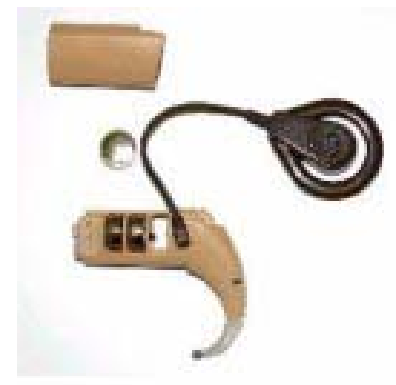

Figure 3. Cochlear implant - retro-auricular model [9].

\section{Auditory Brainstem Implant}

When it is necessary to restore the function hearing in people whose cause of hearing loss does not allow the application of cochlear implants, it is recommendable the auditory brainstem implant, ABI. The alternative to stimulate directly the core of the auditory nerve, located in the brainstem, is possible with this equipment, specifically designed to transmit the sound directly to the brainstem, for patients who do not have the cochlea and the auditory nerve. See Fig. 4.

\section{HEARING AIDS DEVICES}

The amplification of analog signal uses a conventional electronic components that converts the sound wave captured by a microphone into an analogue electrical signal that is amplified in the circuit and filtered and once again, converted into sound waves.

In their use there are some advantages like low price, low power consumption and miniaturization of its components.

The tecnhologie for sound amplification of digital signal uses tens to thousand of transistors that allowes to amplify the acoustic signal much higher than analog technology.

The equipment consists of electronic circuits and transducers, which we call hardware and software that allow controlling digitally circuits with great precision.

Actually, it is possible to find very small hearing aids that can be placed at the bottom of the ear canal, in order to the development of digital technology and the existence of microprocessors.

The background noise is one of the biggest problems for hearing impaired users of hearing aids.

The advanced digital devices are already able to reduce noise and enhance the sounds that are important to understand the speech.

This fact is related to a type of hearing aid which can be programmed to be integrate with all of individual hearing loss.

Within the hearing aids there are external models, including retro-auriculars, prostheses and internal, such as intra-channels.

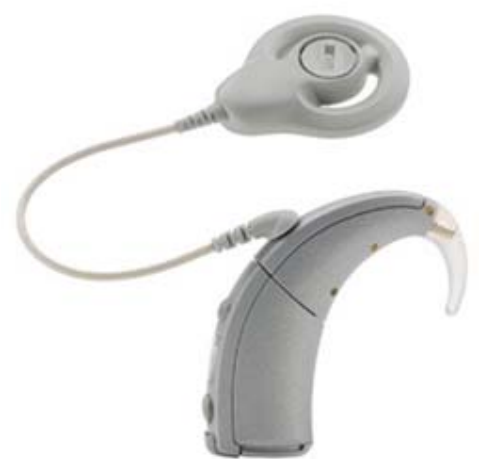

Figure 4. Auditory Brainstem Implant [10].

\section{A. The hearing prostheses}

Behind the ear (BTE), as the name indicates, are located behind the ear, where one tube shaped hook connects the prosthesis to ear mould.

This type of prosthesis is still used in people with severe and deep disabilities, which possibility a large amplification.

This type of prosthesis is usually recommended for children, due to the process of growth heard, because in this type of prosthesis there is only enough mould change, which economically becomes less expensive. See Fig. 5.

\section{B. Bone conduction prostheses}

A large proportion of children with deafness transmission has the option of using conventional prostheses or solves their problems surgically.

However, some of them can be treated surgically but they cannot use conventional prostheses.

This prosthesis amplifies sounds and leads them through an external auditory canal and a middle ear.

On the other way, there are children who were born with microtia or atresia of the external auditory channels that cannot be treated surgically or be fitted with conventional prostheses, designated airway.

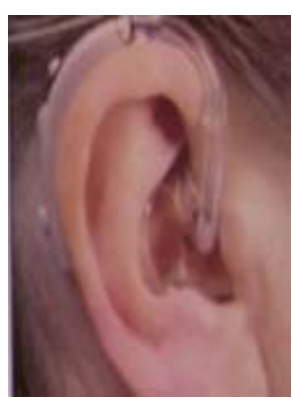

Figure 5. Example of retroauriculars hearing prostheses. 
Therefore, they must use prosthetic bone, which instead of transmitting sound through an order placed on the external auditory bone, transmits the signal to an oscillator, placed on the bone, usually in the mastoid level, which will vibrate and will transmit that vibration to both ears.

The support of these prostheses is achieved through the use of 'bandelettes' or band and stems of glasses, as illustrated in Fig. 6 and 7.

However, the amplification achieved with these devices is not the best, because the use of these prostheses is uncomfortable. It causes local irritation by pressure and also by easily moving.

\section{The intra-chanels hearing}

The intra-channel hearing aids are placed completely inside the ear. It may have different sizes and designations in accordance with the space they occupy and the power they need.

There are three types of hearing intra-channels devices: the in-ear, the intra-canal and intra-deep canal.

The prostheses in the ear (ITE) fill the shell, which is part of the flag auricular or ear, as depicted in Fig. 8.

The prostheses in the canal (ITC) occupy a small part of the flag and one third of auditory channel.

Chosen for aesthetic reasons has the same advantages as the completely in canal (CIC) being easier handling. See Fig. 9.

The completely in canal (CIC) prostheses occupy only the first third of the external ear canal, which fits completely inside the ear canal, being the least visible of the models. Fig. 10.

It is sometimes chosen for aesthetic reasons; acoustically, its advantage is that by being completely inside the ear canal; noise created by wind on the microphone is reduced; its use with the phone is extremely practical.

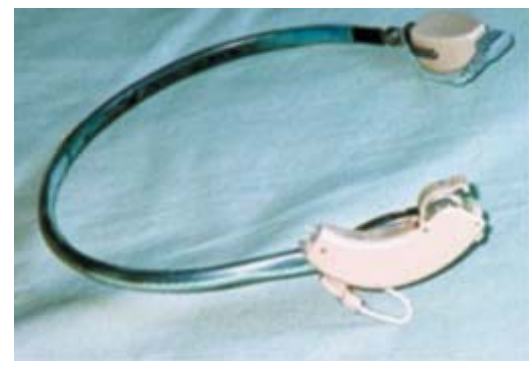

Figure 6. Bone conduction prothesis 'bandelette' [7].

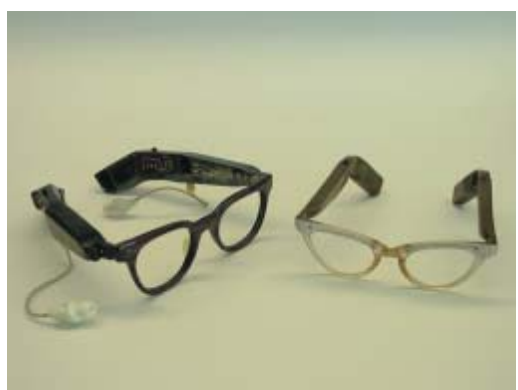

Figure 7. Bone conduction prothesis on stems of glasses [7].

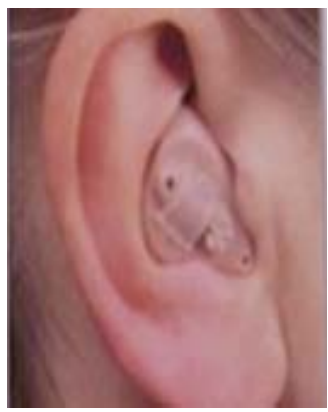

Figure 8. Example intra-auricular hearing prothesis.

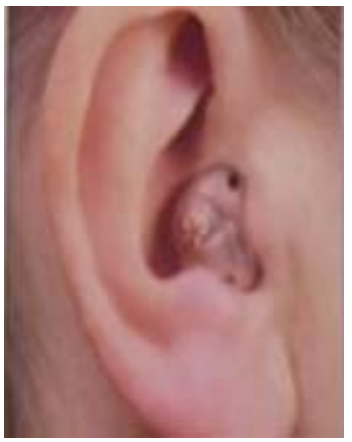

Figure 9. Example intra-chanel hearing prothesis.

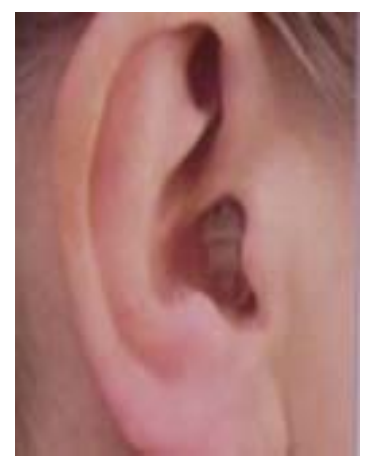

Figure 10. Example deep intra-chanel hearing prothesis.

IV. OTHER TECHNICAL AIDS Not IN THE EAR

In this section other technological useful aids not used close to the ear are presented. 


\section{A. Signal amplifier to the phone}

It is a technology developed with application in telecommunications equipment, such as phone and cell phone, and allows extending 10 times the signal of reception and which can solve problems of lack in the connections. In such way it allows the use of the phone for person with hearing impaired. The amplifier has a key switch that when turned off does not alter the normal operation of phone. The electronic system consist of a circuit linear integrated connected to capacitors, resistors and two transistors, powered by battery under 3 Volts and packed in a box properly scaled [2,3].

\section{B. Amplifier magnetic field to $T V$}

This is very useful in people with hearing impairment who use hearing aids. With this device is possible to capture and inductively transmit the sound of television or phone to an electromagnetic field to which a deaf, with hearing aids turned on, it picks up sound through coil inside the existing unit. Thus, the deaf hear inductively only the sound of television with greater intensity and clarity.

\section{Device optimized for communication between hearing impaired person and public service employee}

This device enables communication between public service employee (listener), who does not know sign language, and hearing impaired in any kind of helpdesk. The equipment allows hearing impaired persons to start the communication by accessing a video screen, or any means of data entry available. It sends images and input data, not sound, that will be translated to a sound, to a listener directly attached to the equipment. The listener sends a sound response to a peripheral device connected to the output translator systems. The system translates the audio signals into written signs, by voice recognition software and a software programme to processing text into sign language, using a sign language dictionary. The signs language, or the text, will appear to the hearing impaired on the computer screen.

\section{Phone with functionality for person with hearing impaired}

By changing the hardware and/or software is possible that cell phone with a handset in the ear, pick up the environment sound and amplified it. Allowing that hearing impaired to use the cell phone for the deafness [2].

\section{E. Bell for the hearing impaired person}

The bell for the hearing impaired person is connected to the terminals of a lamps and outlets. Once the bell is activated causes all the house lights turning on an electronic device that is plugged, causing enough vibration in the bed of the hearing impaired in order to wake him up. The bell is connected to the mains of the residence without damaging the normal operation of the network. The negative phase of the electricity network is connected in the negative phase of the electrical distribution box that connects to the output signal, where the wire is connected to the ringer switch, returning the wire to the distribution box. The positive phase of the electrical network is connected in the positive phase electrical distribution through the connection of the lamps and the sleeves to the outlet. The positive and negative phase output of the electrical distribution box should be connected in positive and negative phase of the sockets of the lamps. For the outlets, the connection should be made separately and only with the electrical distribution box. Every touch the ringer switches the lights flash and the outlets will have electrical power.

\section{CONCLUSIONS}

This paper results from an academic work for describing the available rehabilitation technologies for hearing impairment. The described technologies were grouped in implant devices, prosthesis not implanted and other technical aids not in the ear. Several technologies were described but there were not the objective to exhaust the existing technologies. Therefore some others existing technological aids were not referred.

The rehabilitation is a process that allows a hearing impaired person to have improvement or feel again the sensation of sound. There are several devices that enable deaf improvements in their daily life.

Any professional who works with rehabilitation of individuals with hearing loss, besides having to be constantly updated as to what technology can provide better for your patient, surely we should also ask about the real benefit of that current technology can provide the hearing needs of each individual.

The amplification systems don't repair the injuries that led to hearing loss but may minimize its effects.

\section{REFERENCES}

[1] M. Portmann, "Otorrinolaringologia" Masson, 2a edição, São Paulo, April 1983.

[2] F. John; M. Mike; B. Ramón; T. Stephen, "Use of telecommunication: The needs of people with disabilities", European Cooperation in the field of Scientific and Technical Research COST 219, Madrid, 1989.

[3] G.S. Carlson \& J. Bernstein, "A system for telephone communication between hearing impaired and normal hearing people. The Volta Review, 1986.

[4] Roe, Patrick , "Bridging the Gap? - Acces to Telecommunications for All People", publisheb by Commission of European Communities; 2001

[5] Tetzchner, Stephen; "Issues in Telecommunication and Disability", publisheb by Commission of European Communities; 1991.

[6] http://www.who.int/en// (consultado a 09 de Abril de 2010).

[7] C. Miguel B., "Próteses auditivas osteo-ancoradas", Revista do hospital de crianças Maria Pia: "NASCER E CRESCER", vol. XIII,n³, pp. 201$205,2004$.

[8] http://www.chicagoear.com/implantable hearing_devices/vibrant_sound bridge.htm (consultado a 12 de Abril de 2010).

[9] Y. Bruce W.; S. Anne K., "Segredos em otorrinolaringologia", editora Artmed, Porto Alegre, 1998.

[10] http://www.centroauditivo.com.pt/ (consultado a 09 de Abril de 2010) 